\title{
METILFENIDATO: UNA RECENSIONE
}

\section{ARTICOLO DI REVISIONE}

OLIVEIRA, Vinicius Faustino Lima de ${ }^{1}$, MOREIRA, Danilo José Silva ${ }^{2}$, FONSECA, Juliana Brito da ${ }^{3}$, ROSSI, Karoline ${ }^{4}$, VASCONCELOS, Suzana dos Santos ${ }^{5}$, DIAS, Claudio Alberto Gellis de Mattos ${ }^{6}$, OLIVEIRA, Euzébio de ${ }^{7}$, DENDASCK, Carla Viana ${ }^{8}$, ARAÚJO, Maria Helena Mendonça de ${ }^{9}$, FECURY, Amanda Alves ${ }^{10}$

OLIVEIRA, Vinicius Faustino Lima de. Et. Metilfenidato: una recensione. Revista Científica Multidisciplinar Núcleo do Conhecimento. Anno 06, Ed. 05, Vol. 01, pp. 0514. maggio 2021. ISSN: 2448-0959, Link di accesso: https://www.nucleodoconhecimento.com.br/salute/metilfenidato-una-recensione, DOI: 10.32749/nucleodoconhecimento.com.br/salute/metilfenidato-una-recensione

\section{RIEPILOGO}

Il metilfenidato (MFD) è un medicinale derivato dalle anfetamine che stimola il Sistema Nervoso Centrale (SNC), promuovendo una maggiore attenzione, meno distrazione e un maggiore senso di motivazione. Questo studio ha lo scopo di descrivere le principali caratteristiche farmaceutiche della MFD, evidenziare le indicazioni cliniche per l'uso di

\footnotetext{
${ }^{1}$ Studioso di medicina. Università Federale di Amapá (UNIFAP).

${ }^{2}$ Studioso di medicina. Università Federale di Amapá (UNIFAP).

${ }^{3}$ Accademico medico. Università Federale di Amapá (UNIFAP).

${ }^{4}$ Accademico medico. Università Federale di Amapá (UNIFAP).

${ }^{5}$ Accademico medico. Università Federale di Amapá (UNIFAP).

${ }^{6}$ Biologo, Dottore di Ricerca in Teoria e Comportamento, Professore e ricercatore del Corso di Laurea in Chimica dell'Istituto di Educazione Di Base, Tecnica e Tecnologica di Amapá (IFAP) e del Corso di Laurea in Formazione Professionale e Tecnologica (PROFEPT IFAP).

${ }^{7}$ Biologo, PhD in Malattie Tropicali, Professore e ricercatore del Corso di Educazione Fisica, Università Federale di Pará (UFPA).

${ }^{8}$ Teologo, PhD in Psicoanalisi Clinica. Si occupa da 15 anni di Metodologia Scientifica (Metodo di Ricerca) in Orientamento alla Produzione Scientifica per Master e Dottorandi. Specialista in Ricerche di Mercato e Ricerche Sanitarie Dottorando in Comunicazione e Semiotica (PUC SP).

${ }^{9}$ Dottore, Master in Scienze dell'Insegnamento e della Salute, Professore e ricercatore del Corso di Medicina del Campus Macapá, Università Federale di Amapá (UNIFAP).

${ }^{10}$ Biomedicale, PhD in Malattie Tropicali, Professore e ricercatore del Corso di Medicina del Campus Macapá, Università Federale di Amapá (UNIFAP), Prorettore di Ricerca e Studi Universitari (PROPESPG) dell'Università Federale di Amapá (UNIFAP).
}

$\mathrm{RC}: 84390$

Link di accesso: https://www.nucleodoconhecimento.com.br/salute/metilfenidato-unarecensione 
MFD e dettagliare i possibili eventi avversi derivanti dal consumo di MFD. Per questo, abbiamo condotto una ricerca nei database ScienceResearch.com, SciELO, LILACS, PubMed ed EMBASE e nei repository istituzionali dell'Università Federale di São Paulo, dell'Università Federale di Minas Gerais e dell'Università Federale di Ceará, utilizzando le parole chiave e le associazioni "metilfenidato", "ritalin", "effetti" ed "effects". È stato dimostrato che il farmaco consiste in una miscela racemica formata da quattro stereoisomeri, e il composto farmacologicamente attivo è racemato dextrogyl (d,I)-treo-MFD. Agisce sul blocco dei trasportatori di dopamina e noradrenalina dal SNC, promuovendo effetti che giustificano la sua prescrizione per trattare il disturbo da deficit di attenzione iperattività (TDAH). Negli ultimi anni, il suo consumo per scopi non terapeutici è aumentato. Sono stati riportati alcuni effetti avversi del suo uso e sono stati segnalati insonnia, irritabilità e mal di testa.

Parole chiave: Metilfenidato, Dopamina, Noradrenalina.

\section{INTRODUZIONE}

Il metilfenidato (MFD) è uno stimolatore del sistema nervoso centrale (SNC) della piperidina, un tipo di anfetamina (PAPA, 2013). L'MFD ha un consumo più elevato rispetto alla somma di tutti gli altri psicostimolanti, essendo il più consumato al mondo (SALVIANO, 2015).

Nei primi anni '90, su scala globale, sono state prodotte 2,8 tonnellate di MFD. Nel 2000, questo valore è stato portato a 16 tonnellate e, dieci anni dopo, il punteggio statistico ha raggiunto le 43 tonnellate. In Brasile nel 2011, $413 \mathrm{~kg}$ di MFD (CALIMAN; DOMITROVIC, 2013).

È prescritto per gli individui a cui è stato diagnosticato il disturbo da deficit di attenzione iperattività (TDAH) e per la narcolessia (CALDEIRA, 2015). In Brasile, MFD è disponibile per essere commercializzato con i nomi Ritalina $\AA$, Ritalina LA® e Concerta $\AA$, tutti con assunzione orale. Le dinamiche di rilascio MFD nel corpo hanno opzioni di azione immediate e lente, come Ritalin $\AA^{8}$ che ha effetto immediato, mentre Ritalin $\mathrm{LA} \AA \mathrm{e}$ Concerta $\AA$ presentano formule ad azione lunga (BATISTA, 2015). 
Quando si inizia il trattamento con MFD, le dosi indicate ai pazienti infantili vanno da 5 a $20 \mathrm{mg} /$ giorno, con possibili regolazioni, e gli incrementi possono essere effettuati fino alla dose giornaliera massima di $60 \mathrm{mg} / g i o r n o$. Un'altra opzione per determinare la dose giornaliera è iniziare con due dosi giornaliere di $0,3 \mathrm{mg} / \mathrm{kg} \mathrm{e}$, a seconda delle necessità, un aumento di $0,1 \mathrm{mg} / \mathrm{kg} /$ dose può essere effettuato settimanalmente e non deve superare $2 \mathrm{mg} / \mathrm{kg} / \mathrm{giorno}$. A causa della mancanza di studi rivolti ai bambini sotto i 6 anni di età, l'uso di MFD in questa popolazione non è indicato. Negli adulti, la dose giornaliera di MFD varia da 10 a 72 mg (FARIA, 2017).

L'MFD fornisce maggiore attenzione, con conseguente minore distrazione e maggiore senso di motivazione, portando all'interesse per il raggiungimento di obiettivi e azioni (CÂNDIDO, 2018). In Brasile, I'uso legale della MFD è solo per curare malattie e disturbi. Questo farmaco appartiene all'elenco A3 dei farmaci psicotropi dipendenti da un controllo speciale e la sua acquisizione è condizionata alla speciale ricetta gialla e alla conservazione della prescrizione in farmacia al momento della vendita (FARIA, 2017).

\section{OBIETTIVI}

Descrivere le principali caratteristiche farmaceutiche della MFD. Evidenziare le indicazioni cliniche per l'uso di MFD. Dettagliare i possibili eventi avversi derivanti dal consumo di MFD.

\section{METODI}

I riferimenti utilizzati sono stati ottenuti dalla ricerca nelle banche dati: ScienceResearch.com, SciELO, LILACS, PubMed, EMBASE, Institutional Repository dell'Università Federale di São Paulo, Institutional Repository dell'Università Federale di Minas Gerais e Institutional Repository dell'Università Federale di Ceará, utilizzando le seguenti parole chiave e associazioni: "metilfenidato", "ritalin", "effetti" ed "effects".

II metodo di selezione dei documenti era: identificazione, screening, idoneità e inclusione. Per essere inclusi in questa rassegna, gli studi hanno dovuto avere un testo RC: 84390 Link di accesso: https://www.nucleodoconhecimento.com.br/salute/metilfenidato-una- 
completo accessibile nelle banche dati intervistate, con contenuti pertinenti relativi all'MFD, oltre ad essere disponibili in una delle seguenti lingue: portoghese, inglese o spagnolo. Esclusivamente nella piattaforma PubMed è stato utilizzato un filtro temporale in cui sono state utilizzate le pubblicazioni degli ultimi 5 anni, oltre a utilizzare opere relative all'uomo.

L'identificazione è stata la fine della riunione di studi esistenti nella letteratura sulla MFD nelle banche dati. Lo screening è stato effettuato dopo una rapida analisi del titolo e dell'abstract delle pubblicazioni, elevando questi studi alla categoria di ammissibili. Alla fine, tutte le opere ammissibili sono state pienamente lette e la conseguente esclusione di quelle che non erano allineate agli obiettivi di questa revisione e forse non erano escluse nelle fasi precedenti. Infine, quelli approvati sono stati inclusi in tutte le fasi precedenti del metodo applicato.

\section{RISULTATI}

La ricerca sulla MFD nelle banche dati ha portato a 1038 articoli nella fase metodologica dell'identificazione. Al momento dello screening, ne sono stati esclusi 981 , poiché solo il $5,49 \%$ degli studi era ammissibile. Infine, con la lettura completa di tutti i documenti ammissibili, sono stati inclusi 37 documenti e il 35,09\% di quelli ammissibili è stato scartato.

\section{FARMACOLOGIA MFD}

L'MFD, la cui nomenclatura IUPAC (International Union of Pure and Applied Chemistry) è l'acetato di metile 2-fenil-2 (piperidil), ha due carboni chirali e formula chimica $\mathrm{C}_{14} \mathrm{H}_{19} \mathrm{NO}_{2}$. Consiste in una miscela racemica formata da quattro stereoisomeri, che sono d-treo-MFD, l-treo-MFD, d-erythro-MFD e l-eritro-MFD. II composto farmacologicamente attivo è racemato dextrogyl (d,I)-treo-MFD, che reagisce con i recettori della dopamina nel corpo striato e fornisce stimolazione noradrenergica nella corteccia (BATISTA, 2015; FREESE et al., 2012; LINHARES, 2012). 
MFD è un agonista indiretto di dopamina e noradrenalina, influenzando intensamente I'azione di questi neurotrasmettitori nei centri motori e nell'attenzione (BATISTA, 2015; BATISTELA, 2011). Agisce inibendo la reuptake della dopamina da parte dei neuroni pre-sinaptici delle regioni corticali e subcorticali (FREESE et al., 2012).

\section{APPLICAZIONI TERAPEUTICHE DELLA MFD}

L'MFD è lo psicostimolante di prima linea per l'approccio farmacologico all'TDAH e per il trattamento della Narcolessia, un disturbo del sonno molto raro. Viene utilizzato anche per scopi ricreativi, estetici e cognitivi potenziati legati all'aumento della produttività scolastica e del lavoro (BARROS; ORTEGA, 2011; BATISTELA, 2011; BRANT; CARVALHO, 2012; CALDEIRA, 2015; CÂNDIDO, 2018; SALVIANO, 2015).

È stato riferito che l'associazione della MFD con l'inibitore selettivo della ricattura della serotonina (ISRS) è stata efficace nella terapia dei sintomi della sindrome di Gilles de la Tourette (PASSOS; LÓPEZ, 2010), nonché per il trattamento del deficit cognitivo in un paziente vittima di lesioni cerebrali traumatiche (TCE) (ROCHA; MALLOY-DINIZ; HARA, 2006).

L'MFD è anche usato in pazienti adulti con neoplasie avanzate, al fine di trattare la sonnolenza dovuta all'uso di oppioidi, sintomi depressivi, delirium hipoativo e affaticamento dovuto al cancro. C'è una descrizione che I'MFD è un'alternativa terapeutica per i pazienti con diagnosi di Mania Acuta (CAMPOS et al., 2016; LÓPEZGARCÍA; HEGERL, 2013; PAPA, 2013).

\section{REAZIONI AVVERSE DELL'USO DI MFD}

I bambini e gli adolescenti hanno come principali reazioni avverse l'insonnia e la diminuzione dell'appetito, essendo direttamente proporzionali alle dosi di MFD ingerite, oltre a presentare xerostomia e irritabilità. Può verificarsi un grave coinvolgimento dello stato psichiatrico, potenziando o causando disturbi psicotici o episodi di Mania e Tics. Effetti come dolore addominale e mal di testa sono importanti, così come un piccolo 
aumento della frequenza cardiaca (BATISTA, 2015; FARIA 2017; LOUZÃ; MATTOS, 2007; PASTURA; MATTOS, 2004).

Con l'uso prolungato, effetti collaterali come la riduzione dell'altezza e lo sviluppo della dipendenza chimica nel paziente (BRANT; CARVALHO, 2012; PASTURA; MATTOS, 2004). Inoltre, si osserva che ci possono essere aumenti superiori a $10 \mathrm{mmHg}$ nella pressione sanguigna degli utenti MFD (CÂNDIDO, 2018).

Uno studio che utilizza cavie animali indica che la somministrazione acuta e cronica di MFD danneggia l'acido deossiribonucleico (DNA) nel corpo striato e ippocampo (SALVIANO, 2015). Ci sono rapporti che I'MFD causa anche nausea, vomito diminuzione della crescita e del tasso di peso, aritmie, tosse e allucinazioni, che sono di natura visiva e tattile legate a insetti, reptilils o parassiti (BATISTA, 2015). Nei bambini e nei giovani adulti a cui è stata diagnosticata I'TDAH che usano I'MFD, la psicosi si verifica in 1 persona su 660 (MORAN et al., 2019).

L'alopecia è segnalata per l'uso di MFD (NÚÑES-GARCES; SÁNCHEZ-GAYANGO; ROMERO-PÉREZ, 2018), e che I'MFD a lento rilascio può essere correlato allo sviluppo del Fenomeno Raynaud, un coinvolgimento caratterizzato da un'eccessiva vasocostrizione delle arteriole che irrigano la pelle (OTERO et al., 2012).

Grazina et al. (2018) descrivere la frattura femorale in una ragazza a cui è stato diagnosticato I'TDAH trattato con MFD, associando il farmaco come possibile causa. In questo studio, vengono sottolineati che la densità ossea di un bambino che utilizza MFD è diversa da quella che non usa, suggerendo che il farmaco aumenta il rischio di frattura.

Nei ratti, che hanno tessuto polmonare istologicamente compatibile con l'uomo, la somministrazione di MFD ha causato danni al parenchima polmonare con la distruzione di setti alveolari (RAPELLO et al., 2015). L'MFD può essere considerato un agente induttore di differenziazioni morfologiche nelle ghiandole salivari dei ratti (LIMA et al., 2016). 
L'associazione della MFD allo sviluppo della sclerosi sistemica è descritta in pazienti con sospetta malattia autoimmune e vasopatica (MERIDOR; PRELIEVO, 2019). Ci sono segnalazioni del caso di un paziente di quattro decenni di vita che è stato sottoposto a chirurgia bariatrica con bypass gastrico laparoscopico simile al roux che aveva usato l'MFD per circa un anno, e dopo due settimane di intervento chirurgico ha iniziato a presentare sintomi di tossicità MFD, come paranoia e allucinazioni uditivi (LUDVIGSSON; HAENNI, 2016).

\section{DISCUSSIONE}

\section{MECCANISMO OPERATIVO MFD E SUA METABOLIZZAZIONE}

Sebbene le caratteristiche farmacologiche dell'MFD siano ben consolidate, il suo meccanismo di funzionamento non è ancora pienamente compreso (BRUNELLI, 2013; LINHARES, 2012).

È noto che I'MFD agisce sulle vie dopaminergiche e noradrenergiche delle regioni corticali e subcorticali del cervello, principalmente nel corpo striato, legandosi ai trasportatori di dopamina e noradrenalina, bloccandoli. II risultato è una maggiore disponibilità di questi nelle fessure sinaptiche locali, che genera effetti eccitatori che riflettono sui benefici promossi dall'MFD (BATISTELA, 2011; BRUNELLI, 2013; CANDIDO, 2018; FERNÁNDEZ, 2018; PALHARES, 2015; SALVIANO, 2015).

I principali trasportatori di dopamina affermati sono DAT1 e DRD4. C'è un rapporto in letteratura che alcune mutazioni nei geni che codificano questi trasportatori sono associate a una maggiore risposta all'MFD, una risposta che è anche legata a fattori sociali e clinici (BATISTA, 2015).

L'aumento del livello di dopamina dovuto al farmaco si verifica nelle aree del SNC, che sono suscettibili all'azione dei farmaci di abuso (FARIA, 2017).

La noradrenalina ha NAT come trasportatore, che è il meccanismo primario dell'inattivazione della via dopaminergica. Quando bloccato, aumenta i livelli pre- 
sinaptici di Noradrenalina, con conseguente maggiore attivazione del percorso noradrenegy (LINHARES, 2012).

La dopamina agisce direttamente nella modulazione delle funzioni cognitive e motorie, nel consolidamento della memoria, nella promozione dell'attenzione, oltre all'elaborazione di alcune informazioni ambientali di fondamentale importanza per la sopravvivenza, svolgendo queste funzioni, in parte, sui recettori D1 (funzione eccitatoria) e D2 (funzione inibitrice) della corteccia prefrontale. Pertanto, si presume che il deficit nella sua funzione causi la comparsa di disattenzione e iperattività, due segni clinici compatibili con il quadro dell'TDAH (BATISTELA, 2011; LIANG et al., 2018; LINHARES, 2012; OCHI; VIEIRA, 2016).

In relazione alla noradrenalina, è noto che agisce in aree che influenzano gli stimoli di inibizione comportamentale. Pertanto, si deduce che il deficit nella sua funzione può portare all'incapacità di controllare gli impulsi, un'altra caratteristica dell'TDAH (LIANG et al., 2018; LINHARES, 2012; OCHI; VIEIRA, 2016).

Sul mercato sono disponibili tre formulazioni MFD: Ritalin $®$, Ritalin LA® e Concerta $\mathrm{LA} \Theta$, che variano in relazione all'emifota, al tempo di rilascio e alla durata dell'azione. II primo consiste in una formulazione ad azione rapida, promuovendo picchi MFD intorno a 1-3 ore dopo l'ingestione, con un'emiluità compresa tra 2 e 3 ore e durata dell'azione tra 3 e 6 ore. Gli ultimi due consistono in formulazioni ad azione rapida, che promuovono picchi di MFD intorno alle 3-4 ore dopo l'ingestione, con un'emiluità di 4 ore e una durata dell'azione che varia da 8 a 12 ore. Ciò che differirà da loro è la quantità di dosi che possono essere accosto al giorno: Ritalin LA $\circledast$ può essere assunto 1 o 2 volte al giorno; La Concerta® 1 volta al giorno (LINHARES, 2012; LOUZÃ; MATTOS, 2007; PAPA, 2013).

L'assorbimento dell'MFD avviene completamente e può facilmente attraversare la barriera ematocefalica grazie alla sua liposolubilità (LINHARES, 2012).

Circa 48-96 ore, I'MPH viene metabolizzato dal sistema microsomico epatico, da un processo di idrolisi che genera acido ritalina, substrato eliminato dal corpo per 
escrezione urinaria. II resto del farmaco che non viene metabolizzato verrà eliminato insieme alla secrezione biliare (LINHARES, 2012; PAPA, 2013).

La metabolizzazione dell'MFD nel fegato avviene per azione delle carbossilesterasi (CES) - principalmente CES1. Le mutazioni nel gene che codifica l'enzima sono legate a un deficit nel metabolismo del farmaco, suscettibile l'utente allo sviluppo di reazioni avverse (BATISTA, 2015).

\section{SICUREZZA D'USO}

Poiché si tratta di un derivato delle anfetamine, l'MFD istiga diverse discussioni in letteratura sulla capacità di questo farmaco di causare dipendenza chimica ed uso eccessivo nei consumatori, il che è ancora controverso. La rarità dei casi di dipendenza chimica e abuso suggerisce un basso rischio da parte del metilfenidato (BRANT; CARVALHO, 2012; FERNÁNDEZ, 2018; PALHARES, 2015; PASTURA; MATTOS, 2004).

Si raccomanda di valutare la possibile esistenza di instabilità emotiva nel paziente prima di prescrivere I'MFD, che è dovuto al rischio che questi individui hanno di aumentare spontaneamente le dosi del farmaco (BRANT; OAK, 2012).

Gli individui che hanno avuto in precedenza comorbilità come ipertensione arteriosa sistemica, aritmie cardiache, depressione, epilessia e disturbo bipolare devono essere trattati con cautela per quanto riguarda l'uso di MFD. Nei primi due casi, i pazienti devono essere monitorati nel tempo per rilevare possibili complicanze. Nel terzo caso, mfd è possibile con farmaci antidepressivi. Negli ultimi due casi, viene segnalato che c'è sicurezza per quanto riguarda l'uso di MFD, a condizione che il paziente abbia le sue condizioni cliniche stabilizzate e adeguatamente trattate (LOUZÃ; MATTOS, 2007).

Tra le formulazioni disponibili del farmaco, la formulazione del rilascio prolungato (Concerta $\AA$ ), oltre ad essere la più pratica, perché ha una durata più lunga, è anche la più sicura, poiché previene il verificarsi di effetti dannosi derivanti dall'improvviso 
aumento dei livelli di metilfenidato nel corpo, senza perdere la sua efficacia (FARIA, 2017; LOUZÃ; MATTOS, 2007; PAPA, 2013).

Sebbene la MFD sia inclusa dalla legislazione brasiliana nell'elenco A3 delle sostanze psicotrope, il metilfenidato non ha la stessa farmacotossicità delle altre sostanze di questo gruppo (metanfetamina, phencyclidin, dronabinolo, tra gli altri), il che gli conferisce una maggiore sicurezza d'uso (CARLINI et al., 2003).

\section{CONSUMO DI MFD}

Dal terzo decennio del XX secolo, la classe di farmaci derivati dalle anfetamine ha suscitato interesse da parte delle industrie farmaceutiche per il trattamento dei disturbi comportamentali, in considerazione della sua capacità di stimolare il SNC (CÂNDIDO, 2018; FERNÁNDEZ, 2018).

L'MFD fu sintetizzato nel 1944 da Leandro Panizzon e nel 1954 fu brevettato con il nome di Ritalin (CÂNDIDO et al., 2019). Anche con la sua esistenza, fino ad allora non era noto che il suo uso potesse apportare miglioramenti al quadro dell'TDAH. Questo è stato stabilito solo negli anni ' 60 e '70 quando gli studi hanno dimostrato benefici per i bambini con disturbo comportamentale (BRANT; CARVALHO, 2012; GONÇALVES; PEDRO, 2018).

Nonostante ciò, il consumo di MFD era già stabilito su larga scala in tutto il mondo, essendo utilizzato per trattare l'affaticamento cronico e la narcolessia, oltre ad essere utilizzato per la perdita di peso, il miglioramento delle prestazioni atletiche e le prestazioni intellettuali (BRANT; CARVALHO, 2012; BRUNELLI, 2013). Secondo Barros e Ortega (2011), gli usi non terapeutici sono chiamati ricreativi, quando usati per migliorare il tempo di veglia e la volontà di praticare il tempo libero, le attività estetiche, se usati per la perdita di peso e il miglioramento cognitivo.

Negli anni '60, verificando il miglioramento del quadro clinico dell'TDAH con la somministrazione di MFD, la comunità scientifica ha raggiunto un consenso sull'uso del farmaco per diagnosticare questo disturbo, che è stato inizialmente stabilito per $\mathrm{i}$ 
bambini, ma che, man mano che sono stati sviluppati più studi, è diventato efficace anche per adolescenti e adulti. Se l'individuo con sospetto TDAH ha presentato miglioramenti dopo l'uso dell'MFD, si presumeva che avesse la malattia (BRUNELLI, 2013; Gonçalves; PEDRO, 2018). Questo aspetto, insieme all'ampliamento dei criteri per la diagnosi dell'TDAH e al conseguente aumento dell'incidenza dell'TDAH, hanno contribuito all'esacerbazione del suo consumo negli ultimi anni (GONÇALVES; PEDRO, 2018; SALVIANO, 2015).

Tuttavia, ciò che attira maggiormente l'attenzione nello scenario attuale è l'appropriazione del consumo di MFD a fini non terapeutici, principalmente da parte di individui di età superiore ai 19 anni, studenti del corso, concurseiros, dirigenti, imprenditori e operatori sanitari come principali utenti, essendo la motivazione principale per cercare prestazioni più elevate durante le loro attività (BRANT; CARVALHO, 2012; CALIMAN; RODRIGUES, 2014; CÂNDIDO et al., 2019; GONÇALVES; PEDRO, 2018; MORGAN et al., 2017; SALVIANO, 2015).

Vale la pena evidenziare l'aumento del consumo tra gli adolescenti, per l'uso correlato alla diminuzione dell'appetito e della stanchezza, oltre al neuroenhancement (CÂNDIDO et al., 2019; GONÇALVES; PEDRO, 2018; SALVIANO, 2015). Lo studio condotto da Cândido et al. (2019) ha rilevato che degli studenti valutati che hanno usato metilfenidato per il neuroenhancement, circa un terzo di loro ha acquisito il farmaco senza prescrizione medica. Gli stessi autori richiamano l'attenzione sulla necessità di intervenire su questa pratica, poiché manca la prova dell'efficacia della MFD a tale scopo.

\section{DOSAGGIO DI MFD E LA SUA RELAZIONE CON GLI EFFETTI AVVERSI DEL FARMACO}

Alcuni studi dimostrano che lo sviluppo di effetti avversi dovuti all'uso di MFD, come l'insonnia e la diminuzione dell'appetito, è condizionato dal dosaggio somministrato, e questi eventi possono tornare indietro nel tempo o con diminuzione del dosaggio 
(BATISTA, 2015; BATISTELA, 2011; LOUZÃ; MATTOS, 2007; PASTURA; MATTOS, 2004).

Holmskov et al. (2017), sulla base di uno studio sugli eventi avversi gastrointestinali durante il trattamento con metilfenidato, non ha riscontrato differenze nel rischio di effetti avversi se considerata dose, tipo o durata della somministrazione.

Viene contattata una bassa frequenza di sviluppo di effetti avversi nei consumatori del farmaco e nella maggior parte dei casi sono lievi o moderati (SALVIANO, 2015).

Nel caso dei bambini, il trattamento per l'TDAH viene eseguito con dosaggi da 5 a 20 mg / die, con regolazioni secondo necessità (FARIA, 2017).

$\mathrm{Papa}^{1}$ sottolinea che non si riscontrano cambiamenti cardiovascolari con dosaggi inferiori a $30 \mathrm{mg}$. Questo è importante per la terapia della malattia, poiché ci sono segnalazioni di alterazioni cardiovascolari con l'uso di MFD (FARIA, 2017; HENNISSEN et al., 2017; SALVIANO, 2015).

Per alleviare i disagi generati da alcuni effetti avversi, ci sono raccomandazioni da seguire: interrompere l'uso di MFD con l'emergere di eventuali eventi avversi, al fine di verificare se il farmaco fosse l'agente causale. In caso di peggioramento coincidente con i livelli massimi di MFD nel corpo, si può proporre l'uso della formulazione a rilascio lungo. In insonnia, evitare l'uso di MFD vicino alla buonanotte, aumentare il dosaggio al mattino e diminuire di notte, sono misure efficaci. Per la perdita di appetito, il paziente può optare per la formulazione a breve azione, al fine di gestire il tempo di azione del farmaco e, di conseguenza, l'effetto collaterale promosso da esso (PASTURA; MATTOS, 2004).

\section{CONCLUSIONE}

Gli effetti dell'MFD derivano dall'inibizione dei recettori dei neurotrasmettitori come dopamina e noradrenalina nelle regioni corticali e subcorticali legate al controllo 
motorio e all'attenzione. Con questa inibizione, i neurotrasmettitori sono in maggiore quantità della fessura sinaptica, causando gli effetti del farmaco.

L'MFD può generare dipendenza chimica, in quanto deriva dalle anfetamine, ma in letteratura ci sono pochi casi di dipendenza derivanti dal suo uso.

L'MFD è indicato per i pazienti con diagnosi di TDAH, ma può anche essere utilizzato per altri scopi, tra cui il trattamento della narcolessia, il miglioramento cognitivo, la ricreazione, l'estetica, un approccio alternativo alla mania acuta e il trattamento dei sintomi dei pazienti con neoplasie avanzate.

L'uso di MFD può causare effetti collaterali, i principali dei quali sono l'insonnia e la mancanza di appetito, e questi effetti sono direttamente proporzionali alle dosi ingerite.

La letteratura mostra che la MFD è efficiente nel trattamento dell'TDAH, ma non chiarisce se causa dipendenza, né è influenzata negativamente dalla crescita e dall'aumento di peso dei bambini.

\section{RIFERIMENTI}

BARROS, D.; ORTEGA, F. Metilfenidato e Aprimoramento Cognitivo Farmacológico: representações sociais de universitários. Saúde Soc, v. 20, n. 2, p. 350-63, 2011.

BATISTA, J. M. N. Estudo farmacoterapêutico e farmacogenético em crianças e adolescentes com transtorno do déficit de atenção e hiperatividade tratados com metilfenidato. Tese de Doutorado - Universidade de Federal do Ceará. Fortaleza, p. 95. 2015.

BATISTELA, S. Efeitos da administração aguda de diferentes doses do metilfenidato sobre a cognição de jovens saudáveis. Tese (Mestre em Ciências) - Universidade Federal de São Paulo. São Paulo, p. 88. 2011.

BRANT, L. C.; CARVALHO, T. R. F. Metilfenidato: medicamento gadget da contemporaneidade. Interface comun saúde educ, v. 16, n. 42, p. 623-36, 2012. 
BRUNELLI, L. F. O Metilfenidato (RITALINA®) na escola: Percepção dos educadores da rede de Ensino fundamental I e II. Dissertação (Mestrado em Ciências Humanas e Sociais Aplicadas) Universidade Federal de Minas Gerais. Belo Horizonte, p. 100. 2013.

CALDEIRA, C. M. P. Frequência do transtorno de déficit de atenção e hiperatividade (TDAH) e os problemas psicológicos associados em crianças e adolescentes. Monografia (Especialista em Neurociências) - Universidade de Federal do Ceará. Fortaleza. p. 64. 2015.

CALIMAN, L. V.; DOMITROVIC, N. Uma análise da dispensa pública do metilfenidato no Brasil: o caso do Espírito Santo. Physis, v. 23, n. 3, p. 879-902, 2013.

CALIMAN, L. V.; RODRIGUES, P. H. P. A experiência do uso de metilfenidato em adultos diagnosticados com TDAH. Psicol estud, v. 19, n. 1, p. 125-134, 2014.

CAMPOS, M. P. O. et al. Fadiga relacionada ao câncer: uma revisão. Rev Assoc Med Bras, v. 57, n. 2, p. 211-219, 2016.

CÂNDIDO, R. C. F. Metilfenidato de liberação imediata para o transtorno do déficit de atenção e hiperatividade (TDAH) em adultos - revisão sistemática. Dissertação (Mestrado em Medicamentos e Assistência Farmacêutica) - Universidade Federal de Minas Gerais. Belo Horizonte, p. 147. 2018.

CÂNDIDO, R. C. F. et al. Perini E, Pádua CM, Junqueira DR. Prevalência e fatores associados ao uso de metilfenidato para neuroaprimoramento farmacológico entre estudantes universitários. Psicol conoc Soc, v. 18, p. 1-7, 2019.

CARLINI, E. A. et al. Metilfenidato: influência da notificação de receita A (cor amarela) sobre a prática de prescrição por médicos brasileiros. Rev Psiquiatr Clín, v. 30, n. 1, p. 11-20, 2003.

FARIA, J. C. M. Desempenho acadêmico de estudantes com diagnóstico de transtorno do déficit de atenção e hiperatividade (TDAH) em uso de 
metilfenidato - revisão sistemática. Dissertação (Mestrado em Medicamentos e Assistência Farmacêutica) - Universidade Federal de Minas Gerais. Belo Horizonte, p. 77.2017.

FERNÁNDEZ, L. C. S. Psicoestimulantes para el TDAH: análisis integral para una medicina basada en la prudencia. Rev Asoc Esp Neuropsiq, v. 38, n. 133, p. 301330. 2018.

FREESE, L. et al. Uso não médico de metilfenidato: uma revisão. Trends psychiatry psychother, v 34, n. 2, p. 110-115, 2012.

GONÇALVES, C. S.; PEDRO, R. M. L. R. "Drogas da Inteligência?”: Cartografando as controvérsias do consumo da Ritalina para o aprimoramento cognitivo. Psicol conoc Soc, v. 8, n. 2, p 71-94, 2018.

GRAZINA, R. et al. Fractura por estrés del cuello del fémur en unaniña hiperactiva tratada con metilfenidato - Reporte de caso. Rev Asoc Argent Ortop Traumatol, v. 83, n. 1, p. 45-49, mar. 2018.

HENNISSEN, L. et al. Cardiovascular Effects of Stimulant and Non-Stimulant Medication for Children and Adolescents with ADHD: A Systematic Review and MetaAnalysis of Trials of Methylphenidate, Amphetamines and Atomoxetine. CNS drugs, v. 31, n. 3, p. 199-215, 2017.

HOLMSKOV, M. et al. Gastrointestinal adverse events during methylphenidate treatment of children and adolescents with attention déficit hyperactivity disorder: A systematic review with meta-analysis and Trial Sequential Analysis of randomised clinical trials. PLos ONE, v. 12, n. 6, p. 1-18, 2017.

LIANG, E. F. et al. The Effect of Methylphenidate and Atomoxetine on Heart Rate and Systolic Blood Pressure in Young People and Adults with Attention-Deficit Hyperactivity Disorder (ADHD): Systematic Review, Meta-Analysis, and MetaRegression. Int J Environ Res Health Public, v. 17, n. 1789, p 1-16, 2018. 
LIMA, K. S. et al. Efeitos do metilfenidato sobre as glândulas salivares maternas de camundongos. Rev Odontol UNESP, v. 45, n. 6, p. 316-321, 2016.

LINHARES, M. I. Estudo da Ritalina ${ }^{\circledR}$ (cloridrato de metilfenidato) sobre o sistema nervoso central de animais jovens e adultos: aspectos comportamentais e neuroquímicos. Dissertação (Mestrado em Farmacologia) Universidade Federal do Ceará. Fortaleza, p. 145. 2012.

LÓPEZ-GARCÍA, P.; HEGERL, U. Tratamiento de la manía aguda com metilfenidato: propuesta terapêutica basada en un nuevo modelo fisiopatológico. Rev psiquiatr salud ment, v. 6, n. 2, p. 93-94, 2013.

LOUZÃ, M. R.; MATTOS, P. Questões atuais no tratamento farmacológico do TDAH em adultos com metilfenidato. J bras psiquiatr, v. 56, n. 1, p. 53-56, 2007.

LUDVIGSSON, M.; HAENNI, A. Methylphenidate toxicity after Roux-en-Y gastric bypass. Surg Obes Relat Dis, v. 12, n. 5, p. 55-57, 2016.

MERIDOR, K.; LEVY, Y. Systemic sclerosis induced by CNS stimulants for ADHD: A case series and review of the literature. Autoimmun Rev, v. 19, n. 1, p. 1-3, 2019.

MORAN, L. V. et al. Psychosis with Methylphenidate or Amphetamine in Patients with ADHD. N Engl J Med, v. 380, n. 12, p. 1128-1138, 2019.

MORGAN, H. L. et al. Consumo de Estimulantes Cerebrais por Estudantes de Medicina de uma Universidade do Extremo Sul do Brasil: Prevalência, Motivação e Efeitos Percebidos. Rev bras educ med, v. 41, n. 1, p. 102-109, 2017.

NÚÑES-GARCES, M.; SÁNCHEZ-GAYANGO, A.; ROMERO-PÉREZ, C. Alopecia reversible secundaria a metilfenidato OROS. Rev colomb psiquiatr. 2018. DOI: 10.1016/j.rcp.2018.09.003.

OCHI, N. O.; VIEIRA, G. L. Efeitos do metilfenidato no desempenho motor de crianças com TDAH. Revista Saúde e Pesquisa, v. 9, n. 1, p. 93-96, 2016. 
OTERO, M. I. et al. Metilfenidato y fenómeno de Raynaud secundario. Semergen, v. 39 , n. 6, p. 330-334, 2012.

PALHARES, J. P. P. "Eu tomo medicamentos para estudar": compreendendo a experiência com metilfenidato entre estudantes universitários. Dissertação (Mestrado em Medicamentos e Assistência Farmacêutica - Universidade de Federal de Minas Gerais. Belo Horizonte, p. 86. 2015.

PAPA, M. P. Uso del metilfenidato en pacientes con cáncer avanzado. Rev Med Urug, v. 29 , n. 1, p. 58-63, 2013.

PASSOS, R. B. F.; LÓPEZ, J. R. R. A. Síndrome de Gilles de la Tourette associada ao transtorno de déficit de atenção com hiperatividade: resposta clínica satisfatória a inibidor seletivo da recaptura de serotonina e metilfenidato. J bras psiquiatr, v. 59, n. 2, p. 160-162, 2010.

PASTURA, G; MATTOS, P. Efeitos colaterais do Metilfenidato. Revista de Psiquiatria Clínica, v. 31, n. 2, p. 100-104, 2004.

RAPELLO, G. V. G. et al. Pulmonary emphysema induced by methylphenidate: experimental study. Sao Paulo Med J, v. 133, n. 2, p. 131-134, 2015.

ROCHA, F. L.; MALLOY-DINIZ, L. F.; HARA, C. Emprego de metilfenidato para o tratamento de déficit cognitivo em paciente com seqüela de traumatismo cranioencefálico. J bras psiquiatr, v. 55, n. 1, p. 78-81, 2006.

SALVIANO, L. H. M. S. Estudo de segurança da Ritalina ${ }^{\circledR}$ (cloridrato de metilfenidato) em animais adultos: aspectos de neurotoxicidade $\mathrm{e}$ nefrotoxicidade. Tese de doutorado - Universidade de Federal do Ceará, Fortaleza, p. 149. 2015.

Inviato: Maggio 2021.

Approvato: Maggio 2021.

RC: 84390

Link di accesso: https://www.nucleodoconhecimento.com.br/salute/metilfenidato-unarecensione 